\title{
Subclinical inflammation in relation to insulin resistance in prediabetic subjects with nonalcoholic fatty liver disease
}

\author{
Israt Ara Hossain', Salima Akter², Farjana Rahman Bhuiyan' ${ }^{1}$, Mijanur Rahman Shah', \\ Mohammad Khalilur Rahman ${ }^{4}$ and Liaquat $\mathrm{Ali}^{i^{*}}$
}

\begin{abstract}
Background: Nonalcoholic fatty liver disease (NAFLD) is a metabolic disease commonly associated with obesity, type 2 diabetes, and inflammation-all features of insulin resistant syndrome. However, very limited data are available regarding the association of subclinical inflammation and insulin resistance with NAFLD in a prediabetic state. We, therefore, conducted the study to assess this relationship among this population.
\end{abstract}

Methods: We studied a cross-sectional analytical design of 140 [male/female, 77/63; age in years (ranges), 45 (25-68)] prediabetic subjects after confirming with $75 \mathrm{~g}$ oral glucose tolerance test. The diagnosis of NAFLD was made by ultrasonic examination of the liver and divided into groups of without NAFLD $(n=63)$ and NAFLD $(n=77)$. All individuals underwent anthropometric and clinical examinations. Among laboratory investigations, serum glucose was estimated by glucose oxidase method, serum lipid profile and liver enzymes were measured by the enzymatic colorimetric method and glycated hemoglobin was measured by high performance liquid chromatography technique. Serum insulin and high sensitivity C reactive protein (hsCRP) were measured by enzyme immunoassay technique. Insulin resistance (HOMA-IR) was calculated by homeostasis model assessment (HOMA).

Results: There was significantly higher levels of hsCRP ( $2.82 \pm 1.60$ vs. $1.39 \pm 0.66 \mathrm{mg} / \mathrm{l}, \mathrm{P}<0.001)$ and HOMA-IR $(4.03 \pm 1.39$ vs. $1.98 \pm 1.04, P<0.001)$ in NAFLD subjects compared to their without NAFLD counterparts. hsCRP [odds ratio $(\mathrm{OR})=5.888,95 \%$ confidence interval $(\mathrm{Cl}) 2.673-12.970, \mathrm{P}<0.001$ ] and $\mathrm{HOMA}-\mathrm{IR}(\mathrm{OR}=4.618,95 \% \mathrm{Cl}$ $2.657-8.024, \mathrm{P}<0.001)$ showed significant determinants of NAFLD after potential confounders of body mass index and triglyceride were adjusted.

Conclusions: Subclinical chronic inflammation and insulin resistance seem to be independent mediators of the association between NAFLD and prediabetes. The data also indicate that the inflammatory condition and insulin resistance are associated with each other and these, in turn, are affected by adiposity and dyslipidemia in prediabetic subjects.

\section{Background}

The entity of 'prediabetes', termed by WHO as 'impaired glucose regulation (IGR)' is now being recognized as a constellation of three different disorders which may have different isolated or combined pathophysiological characteristics. A study from BIRDEM has shown

\footnotetext{
*Correspondence: vc@buhs-edu.org

${ }^{1}$ Department of Biochemistry and Cell Biology, Bangladesh University of Health Sciences, Dhaka, Bangladesh

Full list of author information is available at the end of the article
}

that Bangladeshi prediabetic population may be categorized into three groups based on their pathophysiological mechanism [1]. These are isolated impaired fasting glucose (I-IFG), isolated impaired glucose tolerance (I-IGT) and a combination of both defects (IFG-IGT). Since insulin resistance (IR) is associated with the majority of the subjects in the IGR and since this state is known to be associated with increased cardiovascular morbidity and mortality [2], it is imperative to investigate this group 
for the development of nonalcoholic fatty liver disease (NAFLD).

NAFLD is becoming a major public health problem all over the world with increasing incidence of diabetes mellitus, hypertension, dyslipidemia, hepatic steatosis and metabolic syndrome (MS). The prevalence of the disease is expected to increase worldwide and the trend in developing countries is switching towards western lifestyles. The exact etiology of NAFLD is not yet known, but IR is the key mechanism most commonly associated with the pathogenesis of this disorder. This, in turn, seems to create a linkage between NAFLD and type 2 diabetes (T2DM) by subclinical inflammation as a key factor [3]. Although the role of IR in the relationship between subclinical inflammation and NAFLD among the T2DM subjects is fairly well established [2], however, examining this association in a prediabetic state is limited. One study has been done in an urban population of South India and they showed a higher prevalence of NAFLD (54.5\%) among the T2DM subjects compared to those with prediabetes (IFG or IGT) (33\%), I-IGT (32.4\%), I-IFG (27.3\%) and normal glucose tolerance (NGT) (22.5\%) [4]. However, they did not see the association of IR and subclinical inflammation as a reproducible factor in the development of NAFLD.

The pathogenesis of NAFLD leading to metabolic abnormalities has not been fully elucidated. It is characterized by excessive accumulation of free fatty acid within the liver where they are reesterified with glycerol to produce triglyceride (TG). This lipid abnormality leads to the condition of IR by alterations in insulin receptors of peripheral tissues. Increased deposition of fatty acids within the hepatocytes causes oxidative stress with the release of free radicals leading to ATP depletion, mitochondrial dysfunction and hepatic injury-all contribute to the development of inflammation followed by fibrosis [5]. The hepatic steatosis caused by oxidative stress and their underlying mechanism for the development of inflammation still remains unclear. Recent studies revealed that defective insulin action reduces the lipolysis of hepatic fat by decreasing $\beta$-oxidation which enhances the release of nonesterified fatty acid (NEFA) from adipose tissue so that more NEFA are transported through the bloodstream and taken up by the liver. In a secondary event of hepatic fat accumulation causes oxidative stress and mitochondrial dysfunction thereby releasing of reactive oxygen species (ROS) through lipid peroxidation and the generation of proinflammatory cytokines leading to inflammation [6].

High sensitivity $\mathrm{C}$ reactive protein (hsCRP), a proinflammatory cytokine released during the condition of nonalcoholic steatohepatitis (NASH), also a sensitive marker of systemic inflammation, has been shown to be increased in a hyperglycemic sate. Serum hsCRP levels are elevated in subjects with prediabetes or T2DM. Prior clinical studies showed increased levels of hsCRP as a significant risk factor for the progression of future diabetes [7]. Relationship of inflammatory cytokines with elevated blood glucose levels and the role of IR for this association are available. However, it remains unclear whether a relationship exists between hsCRP and IR with NAFLD in a prediabetic state. Nevertheless, data relating the association of subclinical inflammation with NAFLD in prediabetes is limited, therefore, we hypothesized that a high proportion of prediabetic subjects develop NAFLD and it, in turn, is mediated by subclinical chronic inflammation and IR.

\section{Methods}

\section{Study design and subjects}

A cross-sectional analytical study with group comparison design was conducted during November 2012 to March 2013 in a diabetes care Hospital in Bangladesh (BIRDEM) and a total number of 140 (one hundred and forty) prediabetic subjects were recruited in the study. Diabetes and prediabetes were diagnosed following WHO Group Study criteria [8]. Of the total, upper abdomen ultrasonogram had done and the subjects were divided into 77 without NAFLD and 63 NAFLD groups. We excluded subjects with known liver diseases like viral hepatitis, hepatobiliary diseases, malignancies, inflammatory bowel disease, current medication with lipid and glucose lowering drugs, chronic cardiac, renal and respiratory diseases, stroke, type 1 diabetes, recent change $(\geq 10 \%)$ in body weight and pregnant women. All subjects underwent anthropometric measurements of body weight, height, waist and hip circumference (WC and $\mathrm{HC}$ ), systolic and diastolic blood pressures (SBP and DBP) were measured by standard procedures. Body mass index (BMI) of the subjects was calculated using the formula of $\mathrm{BMI}=$ Weight $(\mathrm{kg}) /$ Height $\left(\mathrm{m}^{2}\right)$.

\section{Biochemical analysis}

After overnight fasting (8-14 h) study subjects underwent fasting blood sampling by venipuncture to assess the biochemical tests including fasting and postprandial serum glucose, glycosylated hemoglobin $\left(\mathrm{HbA}_{1 \mathrm{c}}\right)$, total cholesterol (TC), triglyceride (TG), and high density lipoprotein cholesterol (HDL-c), glutamate oxaloacetate transaminase (GOT), glutamate pyruvate transaminase (GPT), gamma glutamate transaminase (GGT) and alkaline phosphatase (ALP). All tests were measured by standard laboratory methods using a conventional automated analyzer (Dimension ${ }^{\circledR}$ clinical chemistry system, Siemens Healthcare Diagnostics Inc. USA). Low density lipoprotein cholesterol (LDL-c) was calculated by 
Friedwald formula [9]. Serum insulin and hsCRP were determined by ELISA technique using commercial kits (DRG-International, Germany) and their optical density (OD) were measured by ELISA plate reader (Multiscan FC, USA). The inter- and intra-assay coefficient of variation (\% CV) for FSG, insulin and hsCRP were 3.35, 4.33 and $5.12 \%$ and $2.1,3.11$ and $4.01 \%$ respectively. Wintergreen method was used to determine the blood erythrocyte sedimentation rate (ESR) [10]. Homeostatic model assessment insulin sensitivity (HOMA \%S) and pancreatic $\beta$-cell function (HOMA \%B) was derived from fasting values of serum glucose and insulin. Homeostatic model assessment insulin resistance (HOMA-IR) was calculated according to the HOMA model formula: HOMA$\mathrm{IR}=$ fasting insulin $\times$ fasting glucose, divided by 22.5 [11]. Total body fat was determined by bioimpedometry.

\section{Radiological evaluations}

Ultrasound imaging of the liver was carried out by a trained radiologist who was blinded of the aims of the study using a high resolution sonography machine (Philips Ultrasound-Ay-MNT-15 TTK, HDI-4000, Netherland) having a $3.5 \mathrm{MHz}$ linear transducer frequency in fasting state of the subjects to assess the degree of steatosis. The presence of fatty liver was confirmed in the absence of alcohol intake by comparative assessment of echoes brightness arising from the hepatic parenchyma with a high level of liver-kidney differentiation, the entrance of echoes into the deepest part of the liver and clear images of the liver blood vessels. NAFLD evaluation was based on scoring the scale as Grade 0: absent (normal echogenicity), Grade 1: mild steatosis, Grade 2: moderate steatosis, Grade 3: severe steatosis [12].

\section{Statistical analysis}

Data of all parameters were presented as the mean \pm standard deviation (SD) or number as appropriate. Comparison of mean values between two groups was tested using student's unpaired $T$ test. According to the distribution of data, we used the natural logarithmic transformation of the ESR skewed values for statistical analysis $[13,14]$. The sample size was calculated by using the regression model for individual predictors and it depends on the desired power $(l-\alpha)$, significance level $(\alpha)$, the number of predictors and the expected effect sizes. Sampling weights were used by using the formula of $\mathrm{N}>50+8 \mathrm{~m}$, where $\mathrm{m}$ is the number of independent variables (IVs) for testing the multiple correlation and $\mathrm{N}>104+\mathrm{m}$ for testing individual predictors [15]. In our study, there were four IVs (Table 3 ) and the calculated sample number was $50+8(4)=82$ cases and $104+4=108$ cases for testing individual predictors. These calculations were based on significance level of $5 \%$ $(\alpha=0.05)$ and $80 \%$ power $(\mathrm{P}=0.20)$. Pearson's regression curve was done to see the correlation of hsCRP with IR in the study subjects. A multiple linear regression analysis was done to investigate the relationship of HOMA-IR with hsCRP in NAFLD subjects after adjusting the effects of major confounding variables of BMI, WHR, TG, and hsCRP respectively. Multivariate logistic regression was used to predict significant determinants of NAFLD (without NAFLD considered as reference) as the dependent variable and BMI, TG, HOMA-IR and hsCRP as independent variables. To evaluate the effects of HOMA-IR and hsCRP with NAFLD, adjusted odds ratio (ORs) and $95 \%$ confidence interval (CI) estimated by controlling the other significant predictors of NAFLD [16]. A P $<0.05$ was considered as statistically significant. All statistical measures were performed using statistical package for social science (SPSS) for windows version 17.0 (SPSS Inc., Chicago, IL, USA).

\section{Results}

The general characteristics of the prediabetic subjects are shown in Table 1 . Of the 140 subjects, 54 had IFG (38.6 \%, M/F 35/19), 36 had IGT $(25.7 \%, 12 / 24)$ and 50 had IFG-IGT $(35.7 \%, 30 / 20)$ respectively. After liver ultrasound, 63 had NAFLD (45\%,35/28) and 77 had without NAFLD $(55 \%, 42 / 35)$ respectively. Among fatty liver severity, 73 (52.1\%) had grade 0, 50 (35.7\%) had grade 1, 14 (10.0 \%) had grade 2 and $3(2.1 \%)$ had grade 3 steatosis. Subjects with prediabetes had higher levels of hsCRP, ESR and HOMA-IR. Sociodemographic, anthropometric, clinical and biochemical characteristics of the study subjects according to their fatty liver group are shown in Table 2. Prediabetic subjects with NAFLD had significantly higher levels of WC $(\mathrm{P}=0.034)$, WHR $(\mathrm{P}=0.004)$, SBP $(\mathrm{P}=0.011)$, PPSG $(\mathrm{P}=0.042)$, TC $(\mathrm{P}=0.010), \mathrm{TG}(\mathrm{P}=0.047), \mathrm{LDL}-\mathrm{c}(\mathrm{P}=0.014), \mathrm{SGOT}$ $(\mathrm{P}=0.026)$, GGT $(\mathrm{P}=0.003)$, hsCRP $(\mathrm{P}<0.001), \log$ ESR $(\mathrm{P}=0.001)$, FSI $(\mathrm{P}<0.001)$, PPSI $(\mathrm{P}<0.001)$ and HOMA-IR $(\mathrm{P}<0.001)$ compared to without NAFLD group. On the other hand, prediabetic subjects with NAFLD, showed significantly lower levels of HDL-c $(\mathrm{P}=0.024)$, HOMA \%S $(\mathrm{P} \leq 0.001)$ and HOMA \%B $(\mathrm{P}=0.007)$ as compared to their without NAFLD counterparts.

Regression curve analysis showed significant positive correlation of HOMA-IR with hsCRP $\left(\mathrm{r}^{2}=0.034\right.$, $\mathrm{P}=0.030$ ) in the total study subjects (Fig. 1).

Association of HOMA-IR with hsCRP in NAFLD subjects are shown in Table 3. In multiple linear regression analysis, HOMA-IR showed significant positive association with BMI $(\beta=0.294, \mathrm{P}=0.016), \operatorname{WHR}(\beta=0.259$, $\mathrm{P}=0.036)$ and hsCRP $(\beta=0.262, \mathrm{P}=0.034)$ after adjusting the effect of major confounder of TG. 
Table 1 General characteristic of the study subjects

\begin{tabular}{|c|c|}
\hline Parameter & $\begin{array}{l}\text { Prediabetic subjects } \\
(n=140)\end{array}$ \\
\hline \multicolumn{2}{|l|}{$\operatorname{Sex}[n(\%)]$} \\
\hline Male & $77(55.0)$ \\
\hline Female & $63(45.0)$ \\
\hline Age (years) & $45.4 \pm 9.4$ \\
\hline $\mathrm{BMI}\left(\mathrm{kg} / \mathrm{m}^{2}\right)$ & $26.06 \pm 4.51$ \\
\hline WC (cm) & $90.4 \pm 8.2$ \\
\hline $\mathrm{HC}(\mathrm{cm})$ & $96.3 \pm 8.7$ \\
\hline WHR & $0.93 \pm 0.04$ \\
\hline$\% \mathrm{BF}$ & $29.7 \pm 7.4$ \\
\hline $\mathrm{SBP}(\mathrm{mmHg})$ & $120 \pm 23$ \\
\hline $\mathrm{DBP}(\mathrm{mmHg})$ & $82 \pm 20$ \\
\hline \multicolumn{2}{|c|}{ Different subgroups of prediabetes [n (\%)] } \\
\hline IFG & $54(38.6)$ \\
\hline IGT & $36(25.7)$ \\
\hline IFG-IGT & $50(35.7)$ \\
\hline \multicolumn{2}{|c|}{ NAFLD evaluation [n (\%)] } \\
\hline Without NAFLD & $77(55.0)$ \\
\hline With NAFLD & $63(45.0)$ \\
\hline Grade 0 & $73(52.1)$ \\
\hline Grade 1 & $50(35.7)$ \\
\hline Grade 2 & $14(10.0)$ \\
\hline Grade 3 & $3(2.1)$ \\
\hline FSG $(\mathrm{mmol} / \mathrm{l})$ & $5.86 \pm 0.45$ \\
\hline PPSG (mmol/l) & $8.15 \pm 1.51$ \\
\hline $\mathrm{HbA}_{1 \mathrm{c}}(\%)$ & $5.85 \pm 0.56$ \\
\hline $\mathrm{TC}(\mathrm{mg} / \mathrm{dl})$ & $193 \pm 39$ \\
\hline $\mathrm{TG}(\mathrm{mg} / \mathrm{dl})$ & $163 \pm 80$ \\
\hline $\mathrm{HDL}-\mathrm{c}(\mathrm{mg} / \mathrm{dl})$ & $37 \pm 7$ \\
\hline LDL-c (mg/dl) & $122 \pm 35$ \\
\hline GOT (IU/I) & $31 \pm 16$ \\
\hline GPT (IU/I) & $35 \pm 16$ \\
\hline GGT (IU/I) & $32 \pm 14$ \\
\hline ALP (IU/I) & $102 \pm 27$ \\
\hline $\mathrm{HsCRP}(\mathrm{mg} / \mathrm{l})$ & $2.04 \pm 1.37$ \\
\hline $\log$ ESR $(\mathrm{mm} / \mathrm{h})$ & $1.31 \pm 0.22$ \\
\hline $\mathrm{FSI}(\mu \mathrm{IU} / \mathrm{ml})$ & $11.26 \pm 7.14$ \\
\hline PPSI ( $\mu \mid \mathrm{I} / \mathrm{ml})$ & $68 \pm 41$ \\
\hline HOMA \%S & $55 \pm 21$ \\
\hline HOMA \%B & $120 \pm 35$ \\
\hline HOMA-IR & $2.91 \pm 1.58$ \\
\hline
\end{tabular}

Results are expressed as number (percentage), mean $\pm S D$; level of significance was calculated by Student's't'test; $n$ number of subjects

Association of hsCRP and HOMA-IR with NAFLD group considering without NAFLD as reference after adjusting the effects of major confounding variables are shown in Table 4. In binary logistic regression analysis, hsCRP $(\mathrm{OR}=5.888, \mathrm{CI} 2.673-12.970, \mathrm{P}<0.001)$ and
Table 2 Sociodemographic, clinical, anthropometric and biochemical indexes of prediabetic subjects without and with NAFLD

\begin{tabular}{|c|c|c|c|}
\hline Parameter & $\begin{array}{l}\text { Without NAFLD } \\
(\mathrm{n}=77)\end{array}$ & $\begin{array}{l}\text { With NAFLD } \\
(n=63)\end{array}$ & $t / P$ value \\
\hline \multicolumn{4}{|c|}{ Different subgroups of prediabetes [n (\%)] } \\
\hline IFG & $33(42.9)$ & $21(33.3)$ & - \\
\hline IGT & $20(26.0)$ & $16(25.4)$ & - \\
\hline IFG-IGT & $24(31.2)$ & $26(41.3)$ & - \\
\hline \multicolumn{4}{|l|}{ Gender [n (\%)] } \\
\hline Male & $35(55.6)$ & $42(54.5)$ & - \\
\hline Female & $28(44.4)$ & $35(45.5)$ & - \\
\hline Age (years) & $44.2 \pm 9.4$ & $46.1 \pm 9.2$ & $-1.666 / 0.098$ \\
\hline BMI $\left(\mathrm{kg} / \mathrm{m}^{2}\right)$ & $25.54 \pm 4.75$ & $26.68 \pm 4.16$ & $-1.489 / 0.139$ \\
\hline WC (cm) & $89 \pm 8$ & $92 \pm 8$ & $-2.137 / 0.034$ \\
\hline $\mathrm{HC}(\mathrm{cm})$ & $95 \pm 9$ & $97 \pm 8$ & $-1.038 / 0.301$ \\
\hline WHR & $0.93 \pm 0.04$ & $0.94 \pm 0.04$ & $-2.098 / 0.038$ \\
\hline$\% B F$ & $29.1 \pm 7.2$ & $30.5 \pm 7.6$ & $-1.094 / 0.276$ \\
\hline SBP (mmHg) & $115 \pm 18$ & $126 \pm 25$ & $-2.899 / 0.004$ \\
\hline $\mathrm{DBP}(\mathrm{mmHg})$ & $78 \pm 18$ & $87 \pm 20$ & $-2.562 / 0.011$ \\
\hline FSG (mmol/l) & $5.88 \pm 0.49$ & $5.84 \pm 0.41$ & $0.534 / 0.594$ \\
\hline PPSG (mmol/l) & $7.92 \pm 1.51$ & $8.44 \pm 1.47$ & $-2.049 / 0.042$ \\
\hline $\mathrm{HbA}_{1 \mathrm{c}}(\%)$ & $5.81 \pm 0.62$ & $5.89 \pm 0.47$ & $-0.879 / 0.381$ \\
\hline TC (mg/dl) & $185 \pm 37$ & $202 \pm 40$ & $-2.603 / 0.010$ \\
\hline TG (mg/dl) & $151 \pm 72$ & $177 \pm 88$ & $-1.925 / 0.047$ \\
\hline $\mathrm{HDL}-\mathrm{c}(\mathrm{mg} / \mathrm{dl})$ & $38 \pm 7$ & $35 \pm 7$ & $2.282 / 0.024$ \\
\hline LDL-c (mg/dl) & $116 \pm 32$ & $130 \pm 36$ & $-2.488 / 0.014$ \\
\hline GOT (IU/I) & $28 \pm 12$ & $34 \pm 18$ & $-2.255 / 0.026$ \\
\hline GPT (IU/I) & $33 \pm 16$ & $37 \pm 17$ & $-1.780 / 0.077$ \\
\hline GGT (IU/I) & $28 \pm 14$ & $36 \pm 13$ & $-3.059 / 0.003$ \\
\hline ALP (IU/I) & $103 \pm 27$ & $100 \pm 26$ & $0.516 / 0.606$ \\
\hline $\mathrm{HsCRP}(\mathrm{mg} / \mathrm{l})$ & $1.39 \pm 0.66$ & $2.82 \pm 1.60$ & $-7.098 /<0.001$ \\
\hline $\log$ ESR (mm/h) & $1.25 \pm 0.20$ & $1.38 \pm 0.22$ & $-3.393 / 0.001$ \\
\hline $\mathrm{FSI}(\mu \mathrm{IU} / \mathrm{ml})$ & $6.83 \pm 2.68$ & $16.68 \pm 7.17$ & $-11.132 /<0.001$ \\
\hline PPSI ( $\mu \mid \mathrm{U} / \mathrm{ml})$ & $55 \pm 38$ & $83 \pm 40$ & $-4.294 /<0.001$ \\
\hline HOMA \%S & $59 \pm 24$ & $48 \pm 15$ & $3.310 /<0.001$ \\
\hline HOMA \%B & $127 \pm 36$ & $111 \pm 32$ & $2.721 / 0.007$ \\
\hline HOMA-IR & $1.98 \pm 1.04$ & $4.03 \pm 1.39$ & $-9.923 /<0.001$ \\
\hline
\end{tabular}
were found to be significant determinants of NAFLD after adjusting the effects of major confounding variables of BMI and TG respectively.

\section{Discussion}

The study demonstrates the first cross-sectional study where the relationship between subclinical inflammation and IR with NAFLD has been evaluated among Bangladeshi prediabetic subjects. In this study, $44 \%$ 


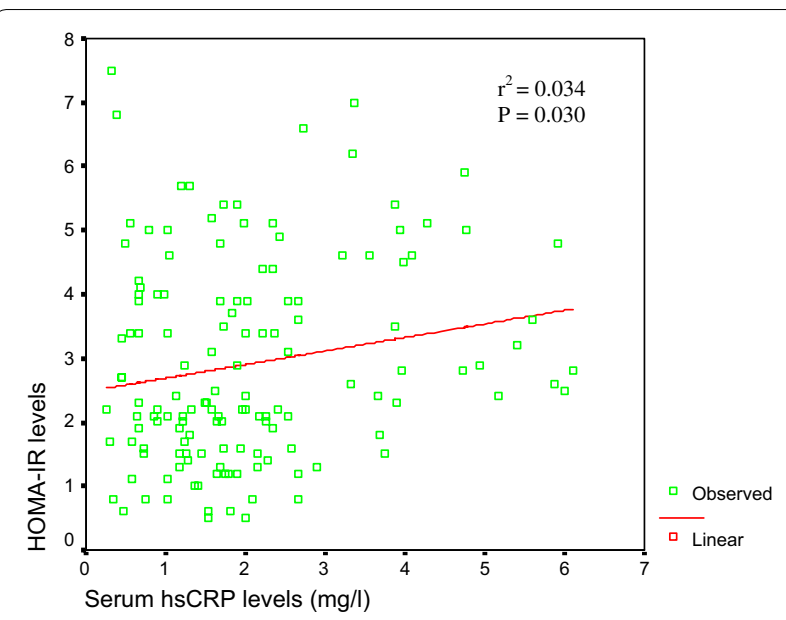

Fig. 1 Relationship of hsCRP with HOMA-IR among the total study subjects: serum hsCRP showed significant positive correlation $(P=0.030)$ with HOMA-IR in the study subjects

Table 3 Multiple linear regression analysis using HOMA-IR as dependent variable after adjusting the effects of major confounders

\begin{tabular}{llrrrr}
\hline Parameter & $\begin{array}{l}\text { Regression } \\
\text { coefficient }(\boldsymbol{\beta})\end{array}$ & t value & P value & \multicolumn{2}{c}{$\begin{array}{l}\text { 95\% Confidence } \\
\text { Interval }\end{array}$} \\
\cline { 4 - 6 } & & & & Lower & Upper \\
\hline $\mathrm{BMl}\left(\mathrm{kg} / \mathrm{m}^{2}\right)$ & 0.294 & 2.483 & 0.016 & 0.019 & 0.178 \\
WHR & 0.259 & 2.144 & 0.036 & 0.564 & 16.449 \\
$\mathrm{TG}(\mathrm{mg} / \mathrm{dl})$ & 0.015 & 0.127 & 0.899 & -0.004 & 0.004 \\
$\mathrm{hsCRP}(\mathrm{mg} / \mathrm{l})$ & 0.262 & -2.167 & 0.034 & -0.440 & -0.017 \\
CONSTANT & - & -1.597 & 0.116 & -13.643 & 1.536 \\
\hline
\end{tabular}

Dependent variable: HOMA-IR, Adjusted $\mathrm{R}^{2}=0.225$; the level of significance at $P<0.05$

of the prediabetic subjects were affected by NAFLD after liver ultrasound scanning which is in accordance with the prior clinical studies $[17,18]$ where prevalence also higher, however, their study subjects were previously detected NAFLD from where the prevalence was calculated. Although conventional view holds that NAFLD is directed related to IR and pre-diabetes, emerging evidence indicate that a direct cause-effect relationship may not exist between these pathological conditions [19]. Nevertheless, very limited data are available regarding the prevalence of NAFLD among the prediabetic subjects. Agarwal et al. [20] showed the prevalence of NAFLD after liver ultrasonography among T2DM subjects was $57.2 \%$. Ruckert et al. [21] found these higher numbers among nondiabetic participants though their fatty liver assessment was based on the abnormal concentration of liver enzymes. They found the fatty liver prevalence in NGT was 18.7 \%, IFG $34.6 \%$, IGT $33.0 \%$, IGT/IFG $33.3 \%$, NDD $42.5 \%$ and known diabetes $25.8 \%$ respectively.

hsCRP is an acute phase reactant protein with a short life of around $18 \mathrm{~h}$, and its level is increased during $\mathrm{NASH}$ causing low-grade systemic inflammation of the liver. Because of its pro-inflammatory characteristics, hsCRP has been considered as a potential biomarker of subclinical inflammation [7]. Recent data from case and control studies showed elevated serum levels of hsCRP in NAFLD subjects and their association also increased with increasing the severity of fatty liver [20, 21]. However, reports from few studies failed to show the relationship of increased levels hsCRP with the different grades of NAFLD. The current study showed higher hsCRP and IR levels in the study subjects and their strong association with NAFLD compared to the without NAFLD group. Our study results are in line with previous cross-sectional studies that revealed a strong association between the hsCRP and NAFLD in Japanese and Korean Asians [22, 23]. A case-control study by Koruk et al. [24] reported that measurement of the degrees of hsCRP could be used as a simple and sensible technique for the diagnosis of NASH and their relationship with histopathological findings of the liver. In addition, Yoneda et al. [25] from their observational studies reported increased levels of hsCRP can be considered the key mechanism of NAFLD development and its rapid progression to NASH due to

Table 4 Factors associated with NAFLD (considering without NAFLD as reference) after adjusting the effects of major confounders by binary logistic regression model

\begin{tabular}{|c|c|c|c|c|c|c|}
\hline \multirow[t]{2}{*}{ Parameter } & \multirow{2}{*}{$\begin{array}{l}\text { Regression } \\
\text { coefficient }(\beta)\end{array}$} & \multirow[t]{2}{*}{ SE } & \multirow[t]{2}{*}{$P$ value } & \multirow[t]{2}{*}{ Odds ratio } & \multicolumn{2}{|c|}{$95 \% \mathrm{Cl}$ of OR } \\
\hline & & & & & Lower & Upper \\
\hline BMI $\left(\mathrm{kg} / \mathrm{m}^{2}\right)$ & 0.108 & 0.069 & 0.117 & 1.114 & 0.973 & 1.276 \\
\hline $\mathrm{TG}(\mathrm{mg} / \mathrm{dl})$ & 0.003 & 0.003 & 0.303 & 1.003 & 0.997 & 1.010 \\
\hline hsCRP (mg/l) & 1.773 & 0.403 & $<0.001$ & 5.888 & 2.673 & 12.970 \\
\hline HOMA-IR & 1.530 & 0.282 & $<0.001$ & 4.618 & 2.657 & 8.024 \\
\hline CONSTANT & -11.331 & 2.577 & 0.000 & 0.000 & - & - \\
\hline
\end{tabular}

SE standard error, $\mathrm{Cl}$ confidence interval

Dependent variable: Group (NAFLD vs. without NAFLD as reference); Adjusted $R^{2}=0.565$; the level of significance at $P<0.05$ 
oxidative stress. Conversely, data from Wieckowska et al. [26] diverge from our previous findings and they found no significant role of hsCRP in the prognosis of NAFLD.

NAFLD is a hepatic manifestation where IR is the key factor due to abnormal lipid metabolism which in turn progress to hepatic injury caused by oxidative stress. The molecular mechanism of IR in the development of NAFLD and the role inflammatory cytokines for their association is controversial. Recent epidemiological evidence reveals IR as a detrimental factor in the pathogenesis of NAFLD and progression to future NASH, particularly in individuals of obese diabetics and related disorders [24]. It has been suggested that genetic factors that reduce insulin sensitivity and increase the accumulation of triacylglycerol levels which ultimately responsible for the development of IR. Our study supports the association between IR and NAFLD as represented by the fact that all the subjects studied are glucose intolerance. Several studies showed the relationship between IR and NAFLD in subjects with diabetes [24-26] however, this is the first study showing the independent roles of IR and subclinical inflammation in the development of NAFLD among Bangladeshi prediabetic subjects.

The abnormal lipid metabolism in the liver due to IR and their consequence in the pathogenesis of NAFLD is not well elucidated, excessive accumulation of triglycerides within the hepatocytes induces the release of ROS resulting oxidative stress, depletion of mitochondrial ATP production, release of pro-inflammatory cytokinesall of these triggers the subclinical chronic inflammation. When hepatocellular damage occurs, macrophage derived inflammatory cytokines (TNF- $\alpha$ and IL-6) are released leading to the production of acute phase reactant protein (hsCRP) [26]. Increased levels of serum hsCRP reveal the manifestation of IR symptoms like obesity, T2DM and cardiovascular morbidity and mortality. Adipose tissue activates the macrophage infiltration thereby releasing IL- 6 and is closely linked to the production of hsCRP, while TNF- $\alpha$ only show its strong relation with IR occurring both in intra-abdominal and subcutaneous tissues $[27,28]$. The pathology of NAFLD in the context of obesity is complicated. It appears that dysregulated adipose remodeling and its subsequent chronic inflammation can be considered as the key etiological factors in the development of fatty liver demonstrated from the experimental observations in ob/ob mice after induction of a high-fat diet $[29,30]$. However, NAFLD itself is a cause or consequence of IR is not well determined. The lipotoxicity effect of liver impairs the insulin action resulting hyperinsulinemia and subsequent leads to the condition of oxidative stress following chronic inflammation and fibrosis. The association between hsCRP and IR with NAFLD also remained consistent by binary logistic regression analysis when other covariates are adjusted, which reinforces the concept that IR is the consequence of hepatic fat accumulation that in turn leads to the development of subclinical inflammation among prediabetic subjects.

The study has several limitations and recommendations. NAFLD examination was done by ultrasonography imaging of the liver but not by liver biopsy which considered as the gold standard method. Correlation between the different stages of NAFLD (by the histologic picture) and the levels of serum inflammatory markers could not be done. Among inflammatory cytokines, the diagnosis of serum hsCRP provides the most promising results revealed by several cross-sectional studies, however, epidemiological study from larger cohort of prediabetes having NAFLD should be considered to reformulate the relationship between serum inflammatory markers with insulin resistance and their causal association with NAFLD. It is an analytical study with a cross-sectional design thus; no causal association between hsCRP and other interacting molecules with NAFLD could be explored.

\section{Conclusions}

From the above study, it may conclude that a high proportion (more than one-third) of the prediabetic subjects has NAFLD and the distribution of the disorder is almost similar in various subgroups of prediabetes. Insulin resistance followed by subclinical chronic inflammation can be considered as key factors in the pathogenesis of NAFLD among prediabetic subjects. The data also indicate that the inflammatory condition and insulin resistance are associated with each other and those, in turn, are affected by adiposity and dyslipidemia in prediabetic subjects.

\section{Abbreviations}

T2DM: type 2 diabetes mellitus; NAFLD: nonalcoholic fatty liver disease; $\mathrm{NASH}$ : nonalcoholic steatohepatitis; $\mathrm{HbA}_{1}$ : glycosylated hemoglobin; HPLC: high performance liquid chromatography; hsCRP: high sensitivity $C$ reactive protein; ELISA: enzyme-linked immunosorbent assay; IGR: impaired glucose regulation; TNF-a: tumor necrosis factor alpha; ROS: reactive oxygen species; $\mathrm{H}_{2} \mathrm{O}_{2}$ : hydrogen peroxide; DNA: deoxyribonucleic acid; BMl: body mass index; WC: waist circumference; HC: hip circumference; WHR: waist to hip ratio; \%BF: percent body fat; SBP: systolic blood pressure; DBP: diastolic blood pressure; IFG: impaired fasting glucose; IGT: impaired glucose tolerance; MS: metabolic syndrome; FSG: fasting serum glucose; PPSG: postprandial serum glucose; TC: total cholesterol; HDL-c: high density lipoprotein-cholesterol; LDL-c: Iow density lipoprotein-cholesterol; GOT: glutamate oxaloacetate transaminase; GPT: glutamate pyruvate transaminase; GGT: gamma glutamate transaminase; ALP: alkaline phosphatase; log ESR: logarithmically transformed erythrocyte sedimentation rate; FSI: fasting serum insulin; PPSI: postprandial serum insulin; HOMA \%S: insulin sensitivity assessed by homeostasis model assessment; HOMA \%B: $\beta$ cell function assessed by homeostasis model assessment; HOMA-IR: homeostasis model assessment insulin resistance. 


\section{Authors' contributions}

The individual contribution of each author's are as follows: IAH: conception and design of the study, acquisition, analysis and interpretation of data, drafting the article; SA, MKR: conception and design of the study; FRB: acquisition of data; MRS: acquisition of data and test analysis; LA: conception and design of the study, supervision, analysis, interpretation of data, drafting the article and revising it critically. All authors read and approved the final manuscript

\section{Author details}

${ }^{1}$ Department of Biochemistry and Cell Biology, Bangladesh University of Health Sciences, Dhaka, Bangladesh. ${ }^{2}$ Department of Biotechnology, Bangladesh University of Health Sciences, Dhaka, Bangladesh. ${ }^{3}$ Department of Microbiology and Immunology, Bangabandhu Sheikh Mujib Medical University (BSMMU), Dhaka, Bangladesh. ${ }^{4}$ Department of Biochemistry and Molecular Biology, University of Dhaka, Dhaka, Bangladesh.

\section{Acknowledgements}

The authors would like to thank all laboratory team members and volunteers, staffs of OPD of BIHS Hospital for their cooperation and helping attitudes and for assisting the conduction of the study.

\section{Availability of data and materials}

The raw data will not be shared due to patient confidentially.

\section{Competing interests}

The authors declare that they have no competing interests.

\section{Consent to publication}

Each participant gave written informed consent prior to study inclusion and to publish their data.

\section{Ethics (consent to participate)}

The study protocol was approved by the ethics committee of Bangladesh Diabetic Association (BADAS). Ref no: BADAS-ERC/13/00106. Subjects were collected from the Out-Patient Department (OPD) of BIRDEM who came for checking their glycemic status. After taking brief history, preliminary selection was done, and the purpose of the study was explained in details to each subject and their verbal consent was taken. All subjects signed written informed consent before participation of the study. Various domains of privacy such as reasonable expectation of individual's privacy (e.g., name, household numbers, educational background, employment history, health history, religion and social status) were removed before analyses to maintain confidentiality.

\section{Funding}

The study was supported partly by Bangladesh Medical Research Counci fund and the award No. 39.012.002.02.01.016.2013-335 from the Government of the People's Republic of Bangladesh, Ministry of Science and Technology.

Received: 2 February 2016 Accepted: 2 May 2016

Published online: 11 May 2016

\section{References}

1. Rahman MH, Hafizur RM, Nahar Q, Khan AR, Ali L. Insulin secretion and sensitivity in Bangladeshi prediabetic subjects. J Diabetes Complicat. 2010:24(1):37-42.

2. Lautamäki R, Borra R, lozzo P, Komu M, Lehtimäki T, Salmi M, et al. Liver steatosis coexists with myocardial insulin resistance and coronary dysfunction in patients with type 2 diabetes. Am J Physiol Endocrinol Metab. 2006:291(2):282-90

3. Angulo P, Hui JM, Marchesini G, Bugianesi E, George J, Farrell GC, et al. The NAFLD fibrosis score: a noninvasive system that identifies liver fibrosis in patients with NAFLD. Hepatology. 2007:45(4):846-54.

4. Mohan V, Farooq S, Deepa M, Ravikumar R, Pitchumoni CS. Prevalence of non-alcoholic fatty liver disease in urban south Indians in relation to different grades of glucose intolerance and metabolic syndrome. Diabetes Res Clin Pract. 2009:84(1):84-91.
5. Neuman G, Sagi R, Shalitin S, Reif S. Serum inflammatory markers in overweight children and adolescents with non-alcoholic fatty liver disease. Isr Med Assoc J. 2010;12(7):410-5.

6. Seppala-Lindroos A, Vehkavaara S, Hakkinen AM. Fat accumulation in the liver is associated with defects in insulin suppression of glucose production and serum free fatty acids independent of obesity in normal men. J Clin Endocrinol Metab. 2002;87(7):3023-8.

7. Utzschneider KM, Kahn SE. The role of insulin resistance in non-alcoholic fatty liver disease. J Clin Endocrinol Metab. 2006;91(12):4753-61.

8. World Health Organization. Definition and diagnosis of diabetes mellitus and intermediate hyperglycaemia: report of a WHO/IDF Consultation. Geneva: World Health Organization. http/www.who.int/diabetes/ publications/diagnosis_diabetes2006

9. Friedewald WT, Levy RI, Fredrickson DS. Estimation of the concentration of low density lipoprotein cholesterol in plasma, without use of the preparative ultracentrifuge. Clin Chem. 1972;18(6):499-502.

10. Jou JM, Lewis SM, Brigys C. ICSH review of the measurement of the erythocyte sedimentation rate. Int J lab Hematol. 2011;33(2):125-32.

11. Matthews DR, Hosker JP, Rudenski AS, Naylor BA, Treacher DF, Turner RC. Homeostasis model assessment: insulin resistance and beta-cell function from fasting plasma glucose and insulin concentrations in man. Diabetologia. 1985:28(7):412-9.

12. Saadeh S, Younossi ZM, Remer EM, Gramlich T, Ong JP, Hurley M, et al. The utility of radiological imaging in nonalcoholic fatty liver disease. Gastroenterology. 2002;123(3):745-50.

13. Bailar JC 3rd, Mosteller F. Guidelines for statistical reporting in articles for medical journals amplifications and explanations. Ann Intern Med. 1988;108(2):266-73.

14. Schor S, Karten I. Statistical evaluation of medical journal manuscripts. JAMA. 1966;195:1123-8

15. Knofczynski GT, Mundfrom D. Sample sizes when using multiple linear regression for prediction. EPM. 2008;68(3):431-42.

16. Altman DG. Statistics in medical journals: developments in the 1980s. Stat Med. 1991;10:1897-913.

17. Gupte P. Non-alcoholic steatohepatitis in type 2 diabetes mellitus. J Gastroenterol Hepatol. 2004;19(8):854-8.

18. Prashanth M, Ganesh HK, Vima MV, John M, Bandgar T, Joshi SR, et al. Prevalence of nonalcoholic fatty liver disease in patients with type 2 diabetes mellitus. J Assoc Physicians India. 2009;57:205-10.

19. Sun Zheng, Lazaremail Mitchell A. Dissociating fatty liver and diabetes. Trends Endocrinol Metab. 2013;24(1):4-12.

20. Agarwal AK, Jain V, Singla S, Baruah BP, Arya V, Yadav R, et al. Prevalence of non-alcoholic fatty liver disease and its correlation with coronary risk factors in patients with type 2 diabetes. J Assoc Physicians India. 2011;59:351-4

21. Ruckert IM, Heier M, Rathmann W, Baumeister SE, Doring A, Meisinger $C$, et al. Association between markers of fatty liver disease and impaired glucose regulation in men and women from the general population: the KORA-F4-Study. PLOS ONE. 2011;6(8):1-11.

22. Riquelme A, Arrese M, Soza A, Morales A, Baudrand R, Pérez-Ayuso RM, et al. Nonalcoholic fatty liver disease and its association with obesity, insulin resistance and increased serum levels of $C$-reactive protein in Hispanics. Liver Int. 2009:29(1):82-8.

23. Park SH, Kim BI, Yun JW, Kim JW, Park DI, Cho YK, et al. Insulin resistance and $\mathrm{C}$-reactive protein as independent risk factors for non-alcoholic fatty liver disease in non-obese Asian men. J Gastroenterol Hepatol. 2004;19(6):694-8.

24. Koruk M, Taysi S, Savas MC, Yilmaz O, Akçay F, Karakök M, et al. Serum levels of acute phase proteins in patients with nonalcoholic steatohepatitis. Turk J Gastroenterol. 2003:14(1):12-7.

25. Yoneda M, Mawatari H, Fujita K, lida H, Yonemitsu K, Kato S, et al. High sensitivity $C$-reactive protein is an independent clinical feature of nonalcoholic steatohepatitis (NASH) and also of the severity of fibrosis in NASH. J Gastroenterol. 2007:42(7):573-82.

26. Wieckowska A, McCullough AJ, Feldstein AE. Noninvasive diagnosis and monitoring of nonalcoholic steatohepatitis: present and future. Hepatology. 2007:46(2):582-9.

27. Kerner A, Avizohar O, Sella R, Bartha P, Zinder O, Markiewicz W, et al. Association between elevated liver enzymes and C-reactive protein: possible hepatic contribution to systemic inflammation in the metabolic syndrome. Arterioscler Thromb Vasc Biol. 2005;25(1):193-7. 
28. Kogiso T, Moriyoshi Y, Shimizu S, Nagahara H, Shiratori K. High-sensitivity C-reactive protein as a serum predictor of nonalcoholic fatty liver disease based on the akaike information criterion scoring system in the general Japanese population. J Gastroenterol. 2009;44(4):313-21.

29. Gao Mingming, Ma Yongjie, Liu Dexi. High-fat diet-induced adiposity, adipose inflammation, hepatic steatosis and hyperinsulinemia in outbred CD-1 mice. PLoS ONE. 2015;10(3):e0119784.
30. Strissel KJ, Stancheva Z, Miyoshi H, Perfield JW, DeFuria J, Jick Z, et al. Adipocyte death, adipose tissue remodeling, and obesity complications. Diabetes. 2007;56(12):2910-8.

\section{Submit your next manuscript to BioMed Central and we will help you at every step:}

- We accept pre-submission inquiries

- Our selector tool helps you to find the most relevant journal

- We provide round the clock customer support

- Convenient online submission

- Thorough peer review

- Inclusion in PubMed and all major indexing services

- Maximum visibility for your research

Submit your manuscript at

www.biomedcentral.com/submit 